\title{
A Left Ventricular Segmentation Method on 3D Echocardiography using Deep Learning and Snake
}

\author{
Suyu Dong ${ }^{1}$, Gongning Luo ${ }^{1}$, Guanxiong Sun, Kuanquan Wang ${ }^{1}$, Henggui Zhang ${ }^{1,2}$ \\ ${ }^{1}$ Harbin Institute of Technology, Harbin, China \\ ${ }^{2}$ University of Manchester, Manchester, UK
}

\begin{abstract}
Segmentation of left ventricular ( $L V$ ) endocardium from $3 D$ echocardiography is important for clinical diagnosis because it not only can provide some clinical indices (e.g. ventricular volume and ejection fraction) but also can be used for the analysis of anatomic structure of ventricle. In this work, we proposed a new full-automatic method, combining the deep learning and deformable model, for the segmentation of $L V$ endocardium. We trained convolutional neural networks to generate a binary cuboid to locate the region of interest (ROI). And then, using ROI as the input, we trained stacked autoencoder to infer the LV initial shape. At last, we adopted snake model initiated by inferred shape to segment the $L V$ endocardium. In the experiments, we used 3DE data, from CETUS challenge 2014 for training and testing by segmentation accuracy and clinical indices. The results demonstrated the proposed method is accuracy and efficiency respect to expert's measurements.
\end{abstract}

\section{Introduction}

Cardiac left ventricular plays a crucial role in the cardiac functions and diagnosis of cardiac disease. Hence, LV volume estimation has attracted much research attention, and segmentation of left ventricular endocardium from 3D echocardiography (3DE) has become a hot topic. Although this is a challenging task, which has to handle many problems inherent in ultrasound imaging, such as low signal-noise ratio, edge dropout and artifacts, researchers have proposed many methods to segment the left ventricular endocardium from 3DE. The typical proposed method can be classified as: deformable models, statistical models and classification methods.

Deformable models are the most common methods of the segmentation of LV endocardium, which translate the problem to an optimization of energy function which is defined by the geometrical constraints. [1] proposed a coupled deformable model which used the speckle statistics information and volume information to form and evolve two surfaces to segment the myocardium. This method can make an automatic segmentation of the full myocardium. Due to intensity distribution of ultrasound tend to Gaussian approximately, [2] combined regionand edge-based level to acquire coarse shape, then adopted this coarse shape as the initial boundary and additional constraint to deform to segment echocardiography. [3] made a motion prior energy as the new constraint of level-set to track the whole myocardium, and used anatomical and image information to adjust hyperparameters. Although many researches have shown deformable models are successful, the models depend much on the initialization and image conditions.

Statistical models, such as active shape model and active appearance model, are based on large labeled information from experts. [4] used a 3D active shape model whose parameters were updated by an extended Kalman filter to segment 3D cardiac ultrasound and the results were promising. [5] proposed a model driven method combining 3D Active Shape Model with local appearance models to segment 3D left ventricular and quantify the left ventricular function. The experiments results suggested the proposed method can achieve acceptable accuracy for the segmentation of fast rotating ultrasound. [6] developed Multiview, multiframe and landmark, and dynamic programming constrained active appearance models. Local edge detector was incorporated into these models. Comparing the experiments results, dynamic programming constrained model is better than the landmark constrained model and active appearance model. Due to depending on a large annotated dataset, initialization and assumption of shape and appearance [7], statistical models are limited for the segmentation of LV.

Classification methods train classifier which is based on different features to segment LV. [8] proposed an automated method and system which used knowledgebased probabilistic model and marginal space learning and can be used to detect standard multiplanar reformatted planes. [9] combined the particle filters with restricted Boltzmann machines achieved competitive 


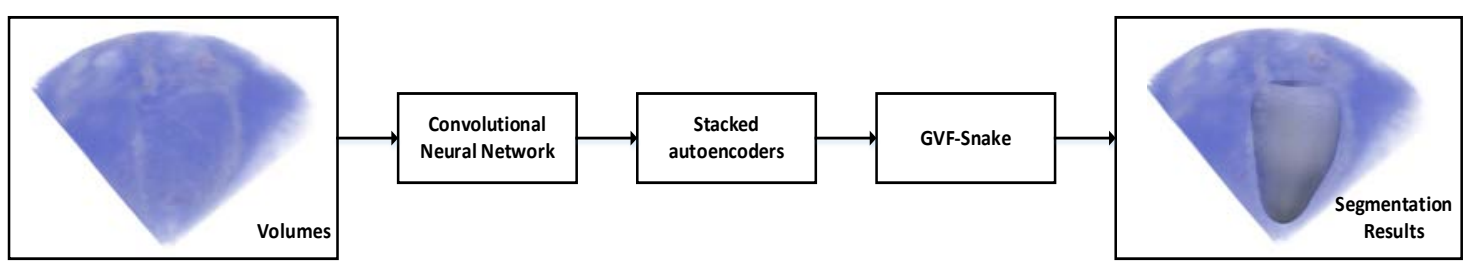

Figure 1 . The proposed method combining the deep learning and deformable model.

tracking results. Analogous to statistical models, classification methods also need a large number of data. However, classification methods do not depend on assumption of shape and appearance.

Recently, deep learning methods are widely used to analyse different images, such as natural images[10] and medical images [11-13], and achieved better results. In this paper, we proposed a full-automatic method for segmentation of the left ventricle from 3DE using deep learning and deformable model. The experiments results show a good agreement with expert's measurements.

\section{Methods}

Inspired by the paper [14], there are three parts of our method combining deep learning and deformable model. As Figure 1 shown, they are the location of the region of interest (ROI) (using convolutional neural network), the inference of LV initial shape (using stacked autoencoders) and segmentation (using GVF-Snake).

\subsection{Location of the region of interest}

Accuracy and efficiency are important for the segmentation algorithm of LV. To improve the accuracy and efficiency of our algorithm, we located the region of interest (ROI).

Due to 3DE data, we acquired a slice from the middle position of the original volumes for every volume firstly. These slices representing original volumes were treated as the inputs of the convolutional neural network. The convolutional neural network contained one convolutional layer, one pooling layer and one fully connected layer. For convolutional layer, there were 16 filters whose size is $31 * 31$. We also adopted the sigmoid as the activation function. Feature maps were acquired by convolved input images. We used maximum pooling to sub-sample the feature maps. The size and stride are $2 * 2$ and 2, respectively. And then, the pooling features were used as the inputs of fully connected layer which had 512 units and achieved logistic regression. At last, we acquired the center of mask. Because of the center of ROI is same with the center of the mask, we cropped a ROI of size $128 * 128$.

Based on the mask, we built a cuboid which size is $128 * 128 * h$. For every volume, $h$ denotes the maximum value of $\mathrm{z}$-axis. This cuboid was used for the location of region of interest in 3D volumes.

\subsection{Inference of $L V$ initial shape}

Deformable models depend much on initialization. So, a good initialization is needed for our segmentation algorithm. In this paper, a stacked autoencoder was used to infer the LV initial shape.

Stacked autoencoder [15] , which treats the outputs of previous layer as the inputs of the next layer, consists of several sparse autoencoders. Figure 2 shows the details of stacked autoencoder. The computation of stacked autoencoder is following:

$$
\begin{gathered}
z^{k}=f\left(w_{k} * a^{k}+b_{k}\right) \\
a^{k+1}=f\left(z^{k+1}\right)
\end{gathered}
$$

Our stacked autoencoder had one input layer, three hidden layer and one output layer. For every ROI, we acquired three slices of volume. We used these slices as the inputs of the stacked autoencoder. Finally, we got three binary masks, which had the corresponding contours of slices of ROI. Based on these contours, we reconstructed initial appearance of ROI.

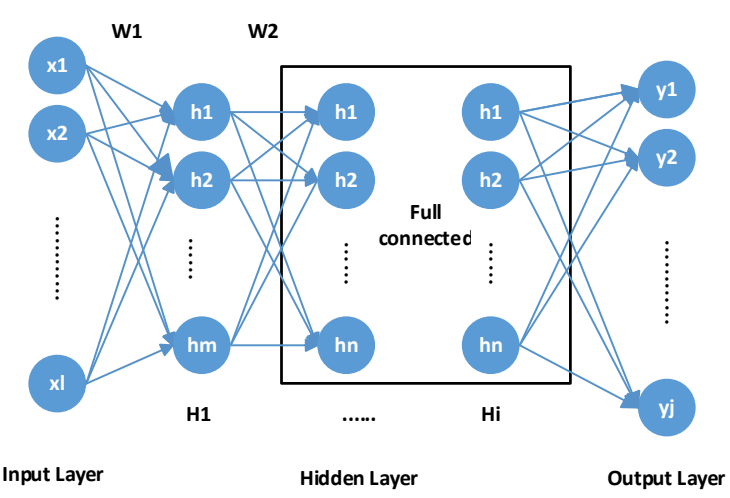

Figure 2. The Structure of Stacked autoencoder.

\subsection{Segmentation}

In this paper, we used three-dimension GVF-snake to segment 3DE. Analogous to two-dimension deformable models, three-dimension GVF-snake is also based on the optimization of energy function. When the energy function achieved the minimum, GVF-snake acquired the three-dimension contours of objects. The three-dimension 
contour and energy function are defined as formula (3) and formula (4), respectively:

$$
\begin{aligned}
X(u) & =[x(u), y(u), z(u)], u=\left(u^{1}, u^{2}\right) \in[0,1] *[0,1] \\
E & =\int \frac{1}{2}\left(\alpha \sum_{i=1}^{2}\left|X_{i}\right|^{2}+\beta \sum_{i, j=1}^{2}\left|X_{i j}\right|^{2}\right)+E_{e x t}(X) d u
\end{aligned}
$$

The first term and second term are internal energy and external energy, respectively. $X_{i}$ and $X_{i j}$ are the firstorder term and second-order term of $X$, and they are controlled by parameter $\alpha$ and $\beta$. The first-order term makes the contour plain and the second-term makes the contour continuous. The energy function is also optimized by gradient descent.

\section{Results}

\subsection{Datasets}

We used 3DE data from CETUS challenge 2014 [16] for training and testing by segmentation accuracy (the mean of mean surface distance $\mathrm{dM}$, hausdorff surface distance $\mathrm{dH}$, modified dice similarity index $\mathrm{D}^{*}$ ). There are 45 sequences of 3D ultrasound volumes, 15 volumes of training set and 30 volumes of test dataset.

\subsection{Metrics}

In term of mean surface distance (dM), hausdorff surface distance $(\mathrm{dH})$, modified dice similarity index $\left(\mathrm{D}^{*}\right)$, we compared the accuracy of our method with the ground truth from cardiologist [16]. $S$ and $S_{t}$ denote the surface from proposed method and surface from ground truth, respectively. Mean surface distance measures the mean distance between $S$ and $S_{t}$, it can be computed by

$$
d_{M}=\frac{1}{2}\left[\bar{d}\left(S, S_{t}\right)+\bar{d}\left(S_{t}, S\right)\right] .
$$

Here, $\bar{d}\left(S, S_{t}\right)$ indicts the mean distance between every voxel from $S$ and the closest voxel from $S_{t}$, the $\bar{d}\left(S_{t}, S\right)$ is computed in similar way. Hausdorff surface distance measures the maximum distance between $S$ and $S_{t}$. Modified dice similarity index measures the overlap of surface and is computed by $D^{*}=\frac{2\left(V \cap V_{t}\right)}{V+V_{t}} . V$ and $V_{t}$ denotes the volume from proposed method and ground truth.

Otherwise, the clinical performance of our method was measured by the modified correlation (corr*) and standard deviation (std) of end-diastolic volumes (EDV), end-systolic volumes (ESV) and ejection fractions. EF is calculated by

$$
E F=\frac{E D V-E S V}{E D V}
$$

And modified correlation is the difference between 1 and correlation.

\subsection{Experiments results and discussion}

Table 1 showed the statistical results of ED and ES of mean surface distance, hausdorff surface distance and modified dice similarity index. These results illustrated that our method can achieve high accuracy. The location of ROI and inference of initial shape improved the accuracy of segmentation. The location of ROI made the influence of the surrounding tissue of segmentation lower. Meanwhile, the location also saved computing time. The

Table 1. Print sizes for different parts of the manuscript.

\begin{tabular}{cccc}
\hline & $\begin{array}{c}\mathrm{dM} \\
(\mathrm{mm})\end{array}$ & $\begin{array}{c}\mathrm{dH} \\
(\mathrm{mm})\end{array}$ & $\begin{array}{c}\mathrm{D}^{*} \\
(\mathrm{val})\end{array}$ \\
\hline ED & 2.20 & 8.34 & 0.112 \\
ES & 2.56 & 8.46 & 0.160 \\
\hline
\end{tabular}

inferred shapes were good initializations of deformable model. They closed to the contours of volumes, which made optimization of deformable model was better and faster. It means that the location of ROI and inference of initial shape improved the accuracy and efficiency, simultaneously.

Figure 3 showed the evaluation of clinical index, which compared the results of the proposed method with the ground truth. The modified correlation values of EDV, ESV and EF are 0.39, 0.40 and 0.267 respectively, and the corresponding standard deviations are $18.2 \mathrm{ml}, 15.7$ $\mathrm{ml}$ and 6.9. The agreement between our method and ground truth is low. However, it also suggested that our method is acceptable and has potential clinical applicability of LV volume estimation. To improve the segmentation accuracy and agreement with the ground truth, larger dataset and modified deformable model are needed. Larger dataset can be used to train and validate deep learning model. In this way, the location of ROI and initial shape will be better. Modified deformable model can provide other constraints which make the contour closer to ground truth.

\section{Conclusion}

In this paper, we proposed a new full-automatic method, combining the deep learning and snake, for the segmentation of LV endocardium. Deep learning was used to locate the region of interest and infer the initial shape. Deformable model which was initialized by the 

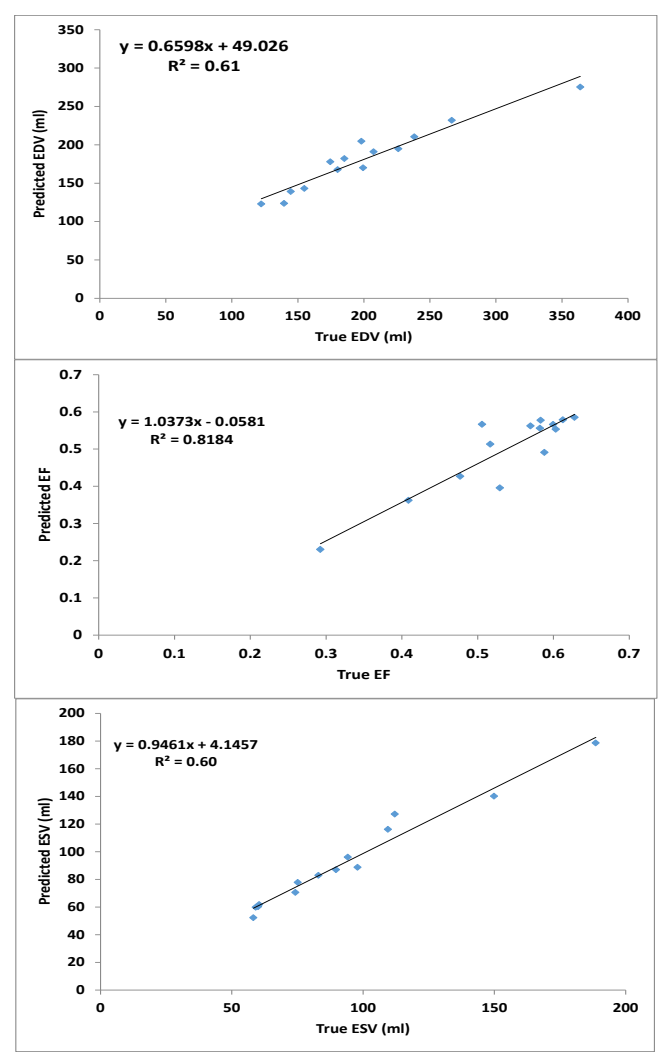

Figure 3. Correlation graphs for EDV, ESV and EF.

inferred shape is used to segment the LV endocardium. Experiments results which were measured by the accuracy and clinical performance demonstrated the proposed method is accuracy and efficiency and respect to expert's measurements.

\section{Acknowledgements}

This work is supported by the National Natural Science Foundation of China (NSFC) under Grant No. 61571165, No. 61572152.

\section{References}

[1] Y. Zhu, X. Papademetris, A. J. Sinusas, and J. S. Duncan, "A coupled deformable model for tracking myocardial borders from real-time echocardiography using an incompressibility constraint," Med Image Anal, vol. 14, pp. 429-48, Jun 2010.

[2] N. Lin, W. Yu, and J. S. Duncan, "Combinative multiscale level set framework for echocardiographic image segmentation," Med Image Anal, vol. 7, pp. 529-37, Dec 2003.

[3] T. Dietenbeck, D. Barbosa, M. Alessandrini, R. Jasaityte, V. Robesyn, J. D'hooge, et al., "Whole myocardium tracking in 2D-echocardiography in multiple orientations using a motion constrained level-set," Medical Image Analysis, vol. 18, pp. 500-514, Apr 2014.
[4] O. F. Hansegård J, Rabben S I.[C]//. Springer Berlin Heidelberg, " Real-time active shape models for segmentation of 3D cardiac ultrasound," presented at the International Conference on Computer Analysis of Images and Patterns, 2007.

[5] M. Ma, M. van Stralen, J. H. Reiber, J. G. Bosch, and B. P. Lelieveldt, "Model driven quantification of left ventricular function from sparse single-beat 3D echocardiography," Med Image Anal, vol. 14, pp. 582-93, Aug 2010.

[6] J. Hansegard, S. Urheim, K. Lunde, and S. I. Rabben, "Constrained active appearance models for segmentation of triplane echocardiograms," IEEE Trans Med Imaging, vol. 26, pp. 1391-400, Oct 2007.

[7] G. Carneiro and J. C. Nascimento, "Combining Multiple Dynamic Models and Deep Learning Architectures for Tracking the Left Ventricle Endocardium in Ultrasound Data," Ieee Transactions on Pattern Analysis And Machine Intelligence, vol. 35, pp. 2592-2607, Nov 2013.

[8] L. X, Georgescu B, Z. Y, and e. al, "AutoMPR: Automatic detection of standard planes in 3D echocardiography," presented at the 2008 5th IEEE International Symposium on Biomedical Imaging: From Nano to Macro., 2008.

[9] N. J. C. Carneiro G, "Multiple dynamic models for tracking the left ventricle of the heart from ultrasound data using particle filters and deep learning architectures," presented at the Computer Vision and Pattern Recognition (CVPR), 2010 IEEE Conference on//. , 2010.

[10] L. Zhang, F. Yang, Y. D. Zhang, and Y. J. Zhu, "Road crack detection using deep convolutional neural network," presented at the IEEE International Conference on Image Processing (ICIP 2016), 2016.

[11] GN Luo, R An, KQ Wang, SY Dong, HG Zhang. A deep learning network for right ventricle segmentation in shortaxis MRI.Computing in Cardiology 2016;43.

[12] GN Luo, GX Sun, KQ Wang, SY Dong, HG Zhang. A novel left ventricular volumes prediction method based on deep learning network in cardiac MRI. Computing in Cardiology 2016;43.

[13] SY Dong, GN Luo, GX Sun, KQ Wang, HG Zhang. A combined multi-scale deep learning and random forests approach for direct left ventricular volumes estimation in 3D echocardiography. Computing in Cardiology 2016;43.

[14] M. R. Avendi, A. Kheradvar, and H. Jafarkhani, "A combined deep-learning and deformable-model approach to fully automatic segmentation of the left ventricle in cardiac MRI," Med Image Anal, vol. 30, pp. 108-19, May 2016.

[15] L. P. Bengio Y, Popovici D, et al, "Greedy layer-wise training of deep networks," Advances in neural information processing systems, p. 19: 153, 2007.

[16] O. Bernard, J. G. Bosch, B. Heyde, M. Alessandrini, D. Barbosa, S. Camarasu-Pop, et al., "Standardized evaluation system for left ventricular segmentation algorithms in 3D echocardiography," IEEE Trans Med Imaging, Nov 252015.

Address for correspondence.

Henggui Zhang

Henggui.zhang@manchester.ac.uk

University of Manchester, Room 3.07 Schuster Building

Manchester, UK, M13 9PL 